\title{
Informationelle Selbstbestimmung am Arbeitsplatz
}
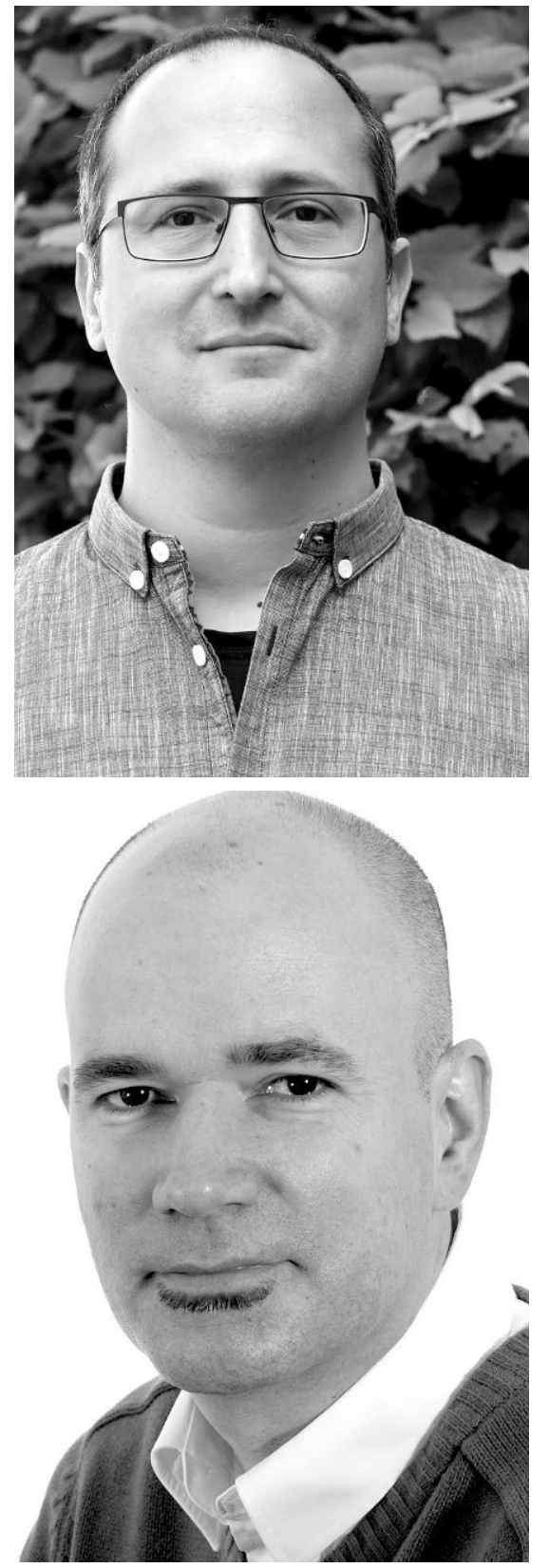

Die Arbeitswelt befindet sich stetig im Wandel. Wie in allen anderen Bereichen des Lebens, führt die Digitalisierung zu den nächsten größeren Umwälzungen im Arbeitsalltag. Dazu zählen nicht nur futuristische Zukunftsszenarien, wie Exoskelette, die Mitarbeiter bei körperlich anstrengenden Tätigkeiten entlasten. Etwas versteckter ermöglicht die Digitalisierung einen bisher nicht gekannten Grad an Optimierung von Geschäftsprozessen und Geschäftsbeziehungen durch eine feingranulare Erhebung, Bereitstellung und Analyse von Daten in Echtzeit. Es gibt viele gute Gründe und nachvollziehbare Unternehmenszwecke, derartige Daten in immer feineren Auflösungen zu verarbeiten, oft auch zum Nutzen der Beschäftigten. Allein die Existenz der Daten weckt jedoch Begehrlichkeiten, diese zweckentfremdet in anderem Kontext zu verwenden. Haben die betreffenden Daten einen Personenbezug, ist dies nicht ohne Weiteres möglich. Den rechtlichen Rahmen geben hier die Datenschutz-Grundverordnung (DSGVO) sowie arbeitsrechtliche Vorschriften vor. Dem Schutz der Privatheit am Arbeitsplatz kommt folglich eine immer größer werdende Bedeutung zu, der aktuell vorwiegend über die Gesetzgebung und Rechtsprechung entsprochen wird.

Um zu einem effektiven Schutz der Privatheit am Arbeitsplatz zu gelangen, mit dem die Beschäftigten ihr Recht auf informationelle Selbstbestimmung tatsächlich ausüben können, braucht es allerdings mehr als nur einen gesetzlichen Rahmen. In diesem Schwerpunktheft möchten wir daher das Thema transdisziplinär beleuchten und hierbei insbesondere die Beschäftigten sowie deren Arbeitgeber in den Mittelpunkt der Betrachtungen stellen.

Die Beiträge in diesem Heft geben einen Überblick über aktuelle Arbeiten zum Thema Beschäftigtendatenschutz - von der Erfassung des aktuellen Ist-Zustandes und Einführung in das Problemfeld, über die Analyse und Bewertung der Rahmenbedingungen, bis hin zu den Wegen für eine effektive praktische Umsetzung.

Aljoscha Dietrich, Christian Bosse und Hartmut Schmitt beleuchten den aktuellen Stand der Kontrolle und Überwachung von Beschäftigten. Vor dem Hintergrund der Digitalisierung weisen sie darauf hin, dass der Gesetzgeber mit detaillierten bereichsspezifischen Regelungen für mehr Klarheit sorgen sollte. Mathias Gisch betrachtet in seinem Beitrag „Führungsinformationssysteme in der öffentlichen Verwaltung - Risiken für die Beschäftigten?" die datenschutzrechtlichen Implikationen IT-basierter Managementunterstützung. Er empfiehlt eine frühzeitige und wirksame Anonymisierung, um datenschutzrechtliche Risiken zu mitigieren. Svenja Polst, Denis Feth, Jan Tolsdorf und Florian Dehling untersuchen in ihrem Beitrag die Datenschutzbedürfnisse von Beschäftigten. Deren Wahrnehmungen und Anforderungen variieren beträchtlich, abhängig vom Wissensstand der Beschäftigten und dem Vertrauen, das sie dem Arbeitgeber entgegenbringen. Christian Bosse, Andreas Weßner und Aljoscha Dietrich zeigen, wie ein Selbstbewertungsinstrument für den betrieblichen Datenschutz die praktische Umsetzung unterstützt. Das Tool hilft dabei, den Status quo im eigenen Unternehmen richtig einzuordnen, zeigt Lücken auf und gibt Handlungsempfehlungen. Hartmut Schmitt und Eduard C. Groen stellen ein Qualitätsmodell zur Förderung des Beschäftigtendatenschutzes vor. Anhand des Modells können IT-Anwendungen aus der Sicht unterschiedlicher Stakeholder bewertet und konfligierende Qualitätsanforderungen aufgedeckt werden. Jan Tolsdorf, Florian Dehling und Denis Feth evaluieren in ihrem Beitrag „Benutzerfreundlicher Datenschutz in cloud-basierten Office-Paketen“ die Datenschutzwerkzeuge gängiger Büroanwendungen. Sie weisen auf schwerwiegende Usabilityprobleme hin und werfen die Frage nach der DSGVO-konformen Ausgestaltung dieser Produkte auf.

Luigi Lo lacono und Hartmut Schmitt 